\section{Mortalidade infantil evitável e fatores associados em Porto Alegre, Rio Grande do Sul, Brasil, 2000-2003}

\author{
Avoidable infant mortality and associated factors \\ in Porto Alegre, Southern Brazil, 2000-2003
}

\begin{abstract}
A case-control study was performed to investigate the association between the categories avoidable and non-avoidable death and sociodemographic, maternal reproductive, and neonatal status. The study used multivariate logistic regression according to a hierarchical model to analyze 1,139 infant deaths from 2000 to 2003. The variables sex, maternal age, number of live born infants, type of pregnancy, place of birth, and 5-minute Apgar were not associated with avoidable deaths. However, maternal schooling $\leq 3$ years ( $R C=1.56$; 95\%CI: 1.01-2.45); single mother ( $R C=0.65$; 95\%CI: 0.49-0.86) or mother with a history of stillbirth $(R C=1.59 ; 95 \% C I$ : 1.01-2.48); congenital malformation $(R C=0.26$; 95\%CI: 0.18-0.37); cesarean section $(R C=1.52$; 95\%CI: 1.10-2.11), premature labor between 22 and 36 weeks ( $R C=0.47 ; 95 \% C I: 0.34-0.65)$, birth weight $<2,500 g(R C=0.51 ; 95 \% C I$ : 0.32 $0.79)$; and neonatal age $<6$ days $(R C=0.62$; 95\%CI: 0.42-0.89) were significantly associated with avoidable death. These findings can help identify infants at increased risk.
\end{abstract}

Infant Mortality; Epidemiologic Factors; Social Conditions
Rita Jobim 1,2

Denise Aerts 1,2

\section{Introdução}

A mortalidade infantil vem diminuindo nos países em desenvolvimento desde o século XX, em razão das melhores condições de vida e saneamento das populações. A partir da década de 60 , avanços tecnológicos na área da saúde e programas, como os de imunizações, aleitamento materno e a rehidratação oral, preconizados pela Organização Mundial da Saúde (OMS), contribuíram para uma forte redução na mortalidade de crianças no primeiro ano de vida 1 .

No Brasil, o coeficiente de mortalidade infantil, que em 1960 foi de 121,1 por mil nascidos vivos 1, diminuiu para 49,4 em 1990 e, em 2002, para 25,1 2. Em Porto Alegre, Estado do Rio Grande do Sul, essa mesma tendência de queda é observada 2, com o coeficiente de 18,4 por mil nascidos vivos em 1996 passando para 13,2 em 2003 3,4. No entanto, as grandes desigualdades existentes na distribuição desses óbitos demonstram que as intervenções em saúde têm beneficiado de forma mais acentuada as crianças de populações mais ricas 5 , e que as crianças de baixa inserção sócio-econômica têm maior dificuldade de acesso aos serviços de saúde e de receberem tratamento adequado 6 .

Essa situação de iniqüidade pode ser revertida com políticas públicas direcionadas às necessidades das populações mais pobres e vulneráveis. Contudo, para o planejamento dessas intervenções, faz-se necessário conhecer a qua- 
lidade da atenção à saúde oferecida. Em 1976, Rutstein et al. 7 propuseram um método clínico de avaliação da qualidade dos serviços de saúde, reconhecendo a saúde como o desfecho da atenção à saúde, quer seja em nível individual ou populacional. Desse modo, o aparecimento de doença, incapacidade e óbito, evitáveis pela utilização de tecnologias em saúde existentes, seria considerado como "evento sentinela" e a sua ocorrência um indicador das potenciais deficiências do sistema de saúde em oferecer o cuidado apropriado e em tempo 8 .

Com o objetivo de avaliar setores específicos da atenção à saúde, alguns autores têm proposto diferentes recortes etários para a classificação de óbitos evitáveis. Charlton et al. 9 excluem as crianças com menos de cinco anos e os maiores de 65 anos, Wigglesworth 10 enfoca o período perinatal, e a classificação utilizada pela Comunidade Européia 11 inclui o primeiro ano de vida somente para determinadas causas de óbito. No Brasil, Ortiz 12, em 1999, propôs uma classificação de evitabilidade para todas as causas básicas de óbito no primeiro ano de vida em categorias sensíveis de redução por intervenções específicas do setor saúde e por parcerias com outros setores, sendo utilizadas pelo Ministério da Saúde como um dos indicadores na avaliação do Pacto da Atenção Básica (http://www.datasus.gov.br/ atbasica/pacto2001, acessado em Out/2003) até o ano de 2003.

O óbito infantil é o resultado de uma extensa cadeia de fatores determinantes ${ }^{5}$. Assim, o conhecimento dos fatores associados aos óbitos evitáveis possibilita planejar intervenções mais adequadas às necessidades dos grupos populacionais em situação de maior vulnerabilidade. Por essa razão, o presente estudo teve o objetivo de investigar a associação entre as categorias óbitos evitáveis e não-evitáveis da classificação de Ortiz e as variáveis sócio-demográficas, reprodutivas maternas e relacionadas às condições de nascimento da criança.

\section{Material e método}

No período de 2000 a 2003, ocorreram 1.180 óbitos de crianças com menos de um ano de idade na população residente em Porto Alegre. Desses, 35 casos foram excluídos por terem sua causa básica considerada como mal definida, portanto sem possibilidade de serem classificados, e seis casos por dados ignorados. Assim, foram analisados, utilizando-se o delineamento transversal, 1.139 óbitos de crianças para os quais foi emitida uma Declaração de Óbito (DO).
As variáveis de interesse foram extraídas de três fontes de dados: Sistema de Informações sobre Mortalidade (SIM), Sistema de Informações sobre Nascidos Vivos (SINASC) e Programa de Vigilância da Morbimortalidade (PRÁ-VIVER) da Secretaria Municipal de Saúde de Porto Alegre. Para a obtenção do desfecho óbito evitável, a variável causa básica de óbito, codificada segundo a Classificação Estatística Internacional de Doenças e Problemas Relacionados à Saúde - 10a Revisão (CID-10) 13, e, após, foi categorizada em evitável e não-evitável utilizando-se a classificação de Ortiz. Os fatores em estudo foram: a idade da criança por ocasião do óbito, coletada do SIM; local de nascimento, sexo, idade gestacional, peso ao nascer, Apgar no 5o minuto, número de filhos nascidos vivos, filhos nascidos mortos, tipo de gravidez, tipo de parto, número de consultas do pré-natal, idade e escolaridade materna, do SINASC e SIM; malformação congênita, do SIM, SINASC e PRÁ-VIVER; estado marital da mãe, do PRÁ-VIVER. Essa última variável foi definida como ter ou não companheiro vivendo sob o mesmo teto com a mãe no momento do óbito da criança.

Por se tratar de um estudo de casos e controles, no qual os óbitos evitáveis foram considerados casos e os óbitos não-evitáveis, como controles, a associação entre o desfecho e os fatores em estudo foi investigada pela técnica da regressão logística, no programa SPSS, versão 10 (SPSS Inc., Chicago, Estados Unidos). Com o objetivo de explorar os dados, foram realizadas análises bivariadas entre o desfecho e cada um dos fatores em estudo, sendo calculadas as razões de chances (RC), os intervalos de confiança (IC95\%) e os níveis de significância.

A análise multivariada foi realizada segundo um modelo conceitual (Figura 1), que estabelece a hierarquia do processo de determinação do desfecho. Essa técnica permite verificar se a associação encontrada entre os fatores em estudo e o desfecho é direta ou mediada por outras variáveis 14,15 .

As variáveis de interesse foram introduzidas em seis etapas. Na primeira, foi analisado o efeito das variáveis sexo da criança e escolaridade materna. A variável sexo da criança foi introduzida nesse primeiro estágio, pois seu efeito sobre o tipo de óbito não é influenciado por outras variáveis. A variável escolaridade materna foi utilizada como um indicador de condições de vida. Para indicar uma associação estatisticamente significativa, foi utilizado um nível de significância de 0,05 e, para que não fossem excluídos potenciais fatores de confusão, um nível de significância $\leq 0,10$ foi utilizado na seleção das variáveis que permaneceram no modelo. Segundo este cri- 


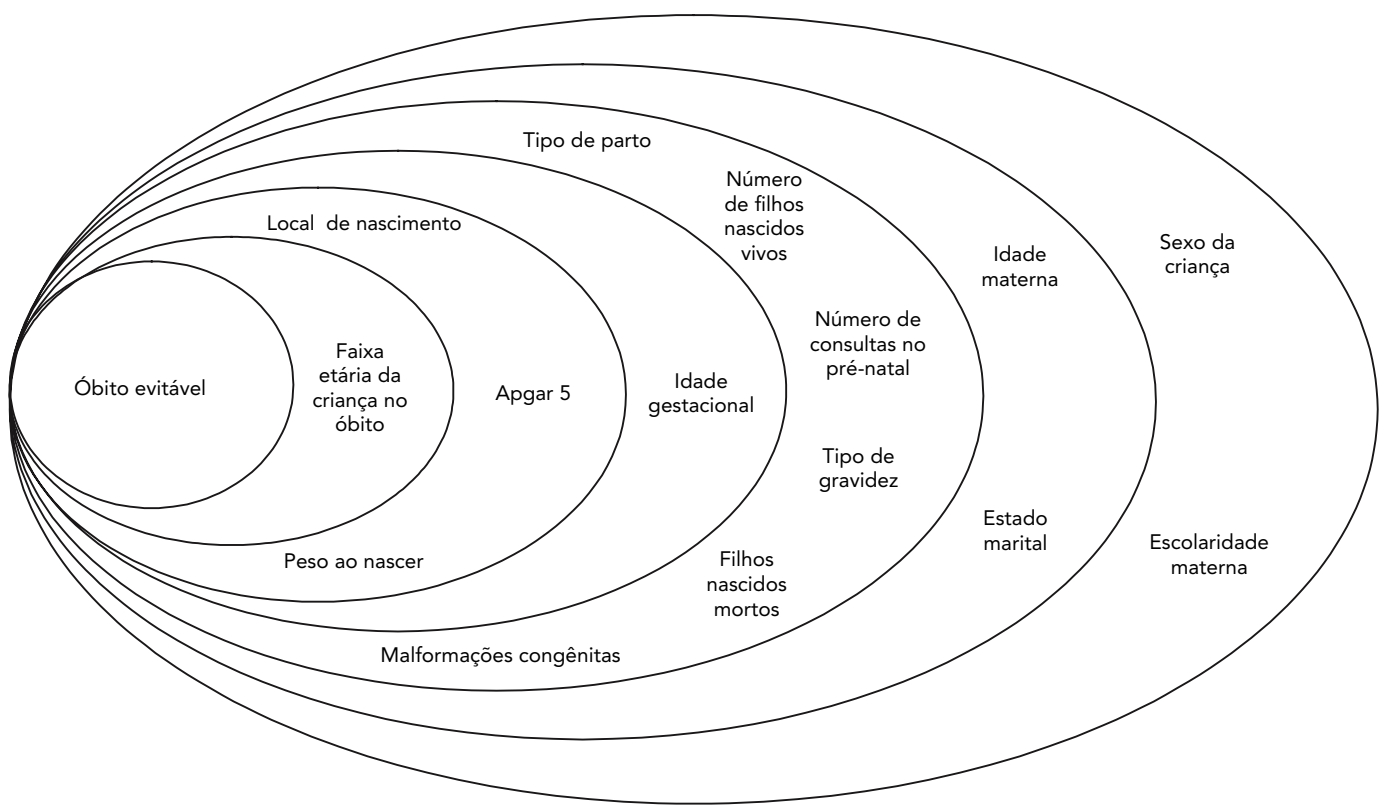

tério, a variável sexo da criança foi mantida no modelo.

$\mathrm{Na}$ segunda etapa, foram introduzidas as variáveis estado marital e idade materna, esta última excluída do modelo. Foram incluídas na terceira etapa as variáveis número de filhos nascidos vivos, filhos nascidos mortos, número de consultas do pré-natal, tipo de gravidez, tipo de parto e malformações congênitas. Número de filhos nascidos vivos, número de consultas do prénatal e tipo de gravidez foram excluídas. Além das variáveis selecionadas anteriormente, foi incluída na quarta etapa a idade gestacional, que permaneceu no modelo. Na quinta etapa, foram introduzidas as variáveis peso ao nascer, local de nascimento e Apgar no 5o minuto, sendo estas duas últimas excluídas. A faixa etária da criança no óbito foi introduzida na sexta etapa.

O modelo final foi composto pelas variáveis escolaridade materna, estado marital, filhos nascidos mortos, malformação congênita, tipo de parto, idade gestacional, peso ao nascer e faixa etária da criança no óbito, que se associaram de forma estatisticamente significativa $(\mathrm{p}<0,05)$ com as suas respectivas razões de chances, intervalo de confiança e valor de p encontrados na etapa em que foram incluídas.
O Comitê de Ética da Universidade Luterana do Brasil (ULBRA) aprovou este estudo (protocolo $\mathrm{n}^{\mathrm{o}}$. 2004-059H), tendo sido considerado sem risco para os participantes por utilizar dados secundários.

\section{Resultados}

\section{Características das crianças}

Entre os 1.139 óbitos estudados, 873 (76,6\%) foram classificados como evitáveis e $266(23,4 \%)$ como não-evitáveis. As características das crianças e de suas mães estão apresentadas na Tabela 1 . Morreram mais meninos do que meninas e a gravidez foi única para $91,7 \%$ das crianças, não tendo sido detectadas malformações congênitas em $83,6 \%$ do total investigado. Houve mais óbitos (59,5\%) de crianças prematuras (22 a 36 semanas). O parto hospitalar foi o mais prevalente $(94,7 \%)$, assim como o vaginal $(60,4 \%)$, sendo que o peso de nascimento foi inferior a $2.500 \mathrm{~g}$ para $58,8 \%$ das crianças e o Apgar no $5^{\circ}$ minuto abaixo de 4 para 12,9\% delas. Em relação à faixa etária da criança no momento do óbito, o período neonatal apresentou $56,9 \%$ do total dos óbitos 
Resultados das análises bivariadas da regressão de logística para o desfecho óbitos evitáveis. Porto Alegre, Rio Grande do Sul, Brasil, 2000-2003.

\begin{tabular}{|c|c|c|c|c|c|c|c|}
\hline \multirow[t]{2}{*}{ Variáveis } & \multicolumn{2}{|c|}{ Óbitos * } & \multicolumn{5}{|c|}{ Óbitos evitáveis } \\
\hline & n & $\%$ & $\mathbf{n}$ & $\%$ & $\mathrm{RC}$ & IC95\% & $\mathbf{p}$ \\
\hline \multicolumn{8}{|l|}{ Sexo } \\
\hline Masculino & 632 & 55,5 & 498 & 78,8 & 1,00 & - & - \\
\hline Feminino & 506 & 44,4 & 375 & 74,1 & 0,77 & $0,58-1,01$ & 0,063 \\
\hline \multicolumn{8}{|l|}{ Tipo de gravidez } \\
\hline Única & 1.045 & 91,7 & 799 & 76,5 & 1,00 & - & - \\
\hline Múltipla & 92 & 8,1 & 72 & 78,3 & 1,11 & $0,66-1,85$ & 0,696 \\
\hline \multicolumn{8}{|l|}{ Malformação congênita } \\
\hline Sim & 187 & 16,4 & 106 & 56,7 & 0,32 & $0,23-0,44$ & 0,000 ** \\
\hline Não & 952 & 83,6 & 765 & 80,6 & 1,00 & - & - \\
\hline \multicolumn{8}{|c|}{ Idade gestacional (semanas) } \\
\hline $22-36$ & 678 & 59,5 & 490 & 72,3 & 0,53 & $0,39-0,71$ & 0,000 ** \\
\hline $37-42$ & 461 & 40,5 & 383 & 83,0 & 1,00 & - & - \\
\hline \multicolumn{8}{|l|}{ Local de nascimento } \\
\hline Hospital & 1.079 & 94,7 & 822 & 78,2 & 1,00 & - & - \\
\hline Outros & 36 & 3,2 & 29 & 80,6 & 1,29 & $0,56-2,99$ & 0,545 \\
\hline \multicolumn{8}{|l|}{ Tipo de parto } \\
\hline Vaginal & 688 & 60,4 & 513 & 74,6 & 1,00 & - & - \\
\hline Cesárea & 451 & 39,6 & 360 & 79,8 & 1,35 & $1,01-1,80$ & 0,041 ** \\
\hline \multicolumn{8}{|c|}{ Peso ao nascer (gramas) } \\
\hline$<2.500$ & 670 & 58,8 & 476 & 71,0 & 0,44 & $0,33-0,60$ & $0,000 * *$ \\
\hline$\geq 2.500$ & 469 & 41,2 & 397 & 84,8 & 1,00 & - & - \\
\hline \multicolumn{8}{|l|}{ Apgar no 5ㅇ minuto } \\
\hline$<4$ & 147 & 12,9 & 111 & 75,5 & 0,41 & $0,63-1,41$ & 0,770 \\
\hline$\geq 4$ & 932 & 81,8 & 714 & 76,6 & 1,00 & - & - \\
\hline \multicolumn{8}{|l|}{ Faixa etária (dias) } \\
\hline $0-6$ & 430 & 37,8 & 299 & 69,5 & 0,44 & $0,32-0,61$ & 0,000 ** \\
\hline $7-27$ & 217 & 19,1 & 162 & 74,6 & 0,57 & $0,39-0,84$ & 0,005 ** \\
\hline $28-364$ & 492 & 43,2 & 412 & 83,7 & 1,00 & - & - \\
\hline \multicolumn{8}{|c|}{ Escolaridade materna (anos) } \\
\hline$\leq 3$ & 173 & 15,2 & 143 & 82,6 & 1,57 & $1,01-2,46$ & 0,048 ** \\
\hline $4-7$ & 514 & 45,1 & 390 & 75,8 & 1,04 & $0,77-1,39$ & 0,772 \\
\hline$\geq 8$ & 452 & 39,7 & 340 & 75,2 & 1,00 & - & - \\
\hline \multicolumn{8}{|l|}{ Idade materna (anos) } \\
\hline$<20$ & 275 & 24,1 & 203 & 74,3 & 0,86 & $0,63-1,18$ & 0,345 \\
\hline$\geq 20$ & 864 & 75,9 & 668 & 77,3 & 1,00 & - & - \\
\hline \multicolumn{8}{|l|}{ Estado marital } \\
\hline Com companheiro & 589 & 51,7 & 473 & 80,3 & 1,00 & - & - \\
\hline Sem companheiro & 542 & 47,6 & 392 & 72,3 & 0,64 & $0,49-0,84$ & 0,002 ** \\
\hline \multicolumn{8}{|c|}{ Número de filhos nascidos vivos } \\
\hline 0 & 306 & 26,9 & 231 & 75,5 & 1,00 & - & - \\
\hline 1 & 416 & 36,5 & 316 & 75,9 & 1,03 & $0,73-1,45$ & 0,884 \\
\hline$\geq 2$ & 415 & 36,4 & 324 & 78,1 & 1,16 & $0,81-1,64$ & 0,416 \\
\hline \multicolumn{8}{|l|}{ Filhos nascidos mortos } \\
\hline Sim & 168 & 14,7 & 140 & 83,3 & 1,63 & $1,06-2,51$ & 0,026 ** \\
\hline Não & 967 & 84,9 & 729 & 83,9 & 1,00 & - & - \\
\hline \multicolumn{8}{|c|}{ Número de consultas no pré-natal } \\
\hline$\leq 3$ & 480 & 42,1 & 361 & 75,2 & 0,83 & $0,59-1,18$ & 0,308 \\
\hline $4-6$ & 349 & 30,6 & 269 & 77,1 & 0,92 & $0,64-1,34$ & 0,683 \\
\hline$\leq 7$ & 292 & 25,6 & 229 & 78,4 & 1,00 & - & - \\
\hline
\end{tabular}

* A soma dos percentuais dos óbitos não totaliza 100\%, em razão de dados ignorados;

** Apresentaram associação estatisticamente significativa. 
infantis, sendo que o período neonatal precoce representou $66,5 \%$ dos óbitos neonatais. A escolaridade de $60,3 \%$ das mães foi de até 7 anos de estudo e 75,9\% delas tinham 20 anos ou mais de idade. No momento do óbito da criança, 51,7\% das mães viviam com companheiro. Na data de nascimento da criança, $72,9 \%$ das mães haviam tido pelo menos um filho nascido vivo e $14,7 \%$ pelo menos um filho nascido morto. Quanto ao pré-natal, $25,6 \%$ das mães fizeram sete ou mais consultas.

As análises bivariadas (Tabela 1) não mostraram associação estatisticamente significativa entre o desfecho e as variáveis sexo, tipo de gravidez, local de nascimento, Apgar no $5^{\circ}$ minuto, idade materna, número de filhos nascidos vivos e número de consultas no pré-natal. Em contrapartida, malformação congênita, idade gestacional, tipo de parto, peso ao nascer, faixa etária, escolaridade materna, estado marital e presença de filhos nascidos mortos se associaram significativamente.

\section{Fatores associados aos óbitos evitáveis}

Na 1ạ etapa da Regressão Logística multivariada (Tabela 2), a variável sexo não apresentou associação significativa. As crianças falecidas por óbitos evitáveis tiveram 1,56 vez mais chance de serem filhas de mães com escolaridade materna menor ou igual a três anos de estudo do que as que faleceram por causas não-evitáveis. Na etapa seguinte, a variável idade materna não mostrou associação significativa. No entanto, diferente dessa, o estado marital associou-se ao desfecho, mostrando que não ter companheiro no momento do óbito do filho esteve associado a uma diminuição da ocorrência dos casos. Foram introduzidas seis variáveis na 3a etapa da análise. Dessas, somente três apresentaram associação com o desfecho ao serem controladas pelo efeito das variáveis selecionadas anteriormente e pelas do mesmo bloco. Entre as crianças que faleceram por óbitos evitáveis verificou-se $59 \%$ e $52 \%$ mais chances de terem, respectivamente, irmãos nascidos mortos e nascidos por parto cesáreo. Em contrapartida, essas crianças tiveram menos chances de apresentarem algum tipo de malformação congênita, isto é, a malformação apresentou um comportamento de proteção para o óbito evitável.

$\mathrm{Na} 4$ a etapa, verificou-se que os casos tiveram menos chances de serem prematuros do que os controles. As variáveis local de nascimento, Apgar no 5 o minuto e peso ao nascer foram introduzidas na $5 \underline{a}$ etapa. Somente essa última associouse ao desfecho, indicando que crianças falecidas por causas evitáveis tiveram menos chances de
Tabela 2

Regressão de logística multivariada para o desfecho óbitos evitáveis, segundo modelo hierarquizado. Porto Alegre, Rio Grane do Sul, Brasil, 2000-2003.

\begin{tabular}{|c|c|c|c|}
\hline \multirow[t]{2}{*}{ Variáveis } & \multicolumn{3}{|c|}{ Óbitos evitáveis } \\
\hline & $\mathrm{RC}$ & IC95\% & $\mathrm{p}$ \\
\hline \multicolumn{4}{|l|}{ 1a Etapa } \\
\hline \multicolumn{4}{|l|}{ Sexo } \\
\hline Masculino & 1,00 & - & - \\
\hline Feminino & 0,77 & $0,59-1,02$ & 0,067 \\
\hline \multicolumn{4}{|c|}{ Escolaridade materna (anos) } \\
\hline$\leq 3$ & 1,56 & $1,01-2,45$ & 0,048 * \\
\hline $4-7$ & 1,05 & $0,78-1,41$ & 0,764 \\
\hline$\geq 8$ & 1,00 & - & - \\
\hline \multicolumn{4}{|l|}{ 2a Etapa } \\
\hline \multicolumn{4}{|l|}{ Idade materna (anos) } \\
\hline$<20$ & 0,37 & $0,70-1,30$ & 0,696 \\
\hline$\geq 20$ & 1,00 & - & - \\
\hline \multicolumn{4}{|l|}{ Estado marital } \\
\hline Com companheiro & 1,00 & - & - \\
\hline Sem companheiro & 0,65 & $0,49-0,86$ & 0,003 * \\
\hline \multicolumn{4}{|l|}{ 3ạ Etapa } \\
\hline \multicolumn{4}{|c|}{ Número de filhos nascidos vivos } \\
\hline 0 & 1,00 & - & - \\
\hline 1 & 1,06 & $0,73-1,52$ & 0,764 \\
\hline$\geq 2$ & 1,12 & $0,76-1,65$ & 0,551 \\
\hline \multicolumn{4}{|l|}{ Filhos nascidos mortos } \\
\hline Sim & 1,59 & $1,01-2,48$ & 0,047 * \\
\hline Não & 1,00 & - & - \\
\hline \multicolumn{4}{|c|}{ Número de consultas no pré-natal } \\
\hline$\leq 3$ & 0,73 & $0,49-1,09$ & 0,126 \\
\hline $4-6$ & 0,84 & $0,56-1,25$ & 0,397 \\
\hline$\geq 7$ & 1,00 & - & - \\
\hline \multicolumn{4}{|l|}{ Tipo de gravidez } \\
\hline Única & 1,00 & - & - \\
\hline Múltipla & 0,98 & $0,57-1,67$ & 0,932 \\
\hline \multicolumn{4}{|l|}{ Malformação congênita } \\
\hline Sim & 0,26 & $0,18-0,37$ & 0,000 * \\
\hline Não & 1,00 & - & - \\
\hline \multicolumn{4}{|l|}{ Tipo de parto } \\
\hline Vaginal & 1,00 & - & - \\
\hline Cesárea & 1,52 & $1,10-2,11$ & 0,010 * \\
\hline \multicolumn{4}{|l|}{ 4ạ Etapa } \\
\hline \multicolumn{4}{|c|}{ Idade gestacional (semanas) } \\
\hline $22-36$ & 0,47 & $0,34-0,65$ & 0,000 * \\
\hline $37-42$ & 1,00 & - & - \\
\hline \multicolumn{4}{|l|}{ 5ạ Etapa } \\
\hline \multicolumn{4}{|l|}{ Local de nascimento } \\
\hline Hospital & 1,00 & - & - \\
\hline Outros & 0,68 & $0,13-3,47$ & 0,639 \\
\hline Peso ao nascer (gram & & & \\
\hline$<2.500$ & 0,51 & $0,32-0,79$ & 0,030 * \\
\hline$\geq 2.500$ & 1,00 & - & - \\
\hline Apgar no 5o minuto & & & \\
\hline$\geq 4$ & 1,00 & - & - \\
\hline$<4$ & 1,24 & $0,79-1,92$ & 0,346 \\
\hline 6a Etapa & & & \\
\hline Faixa etária (dias) & & & \\
\hline $0-6$ & 0,62 & $0,42-0,89$ & 0,010 * \\
\hline $7-27$ & 0,77 & $0,50-1,20$ & 0,250 \\
\hline $28-364$ & 1,00 & - & - \\
\hline
\end{tabular}

* Apresentaram associação estatisticamente significativa. 
apresentarem baixo peso ao nascer do que as que faleceram por causas não-evitáveis. A variável faixa etária foi introduzida na última etapa. Não se encontrou diferenças significativas entre os óbitos ocorridos nos períodos neonatal tardio e pós-neonatal, entretanto, os óbitos evitáveis apresentaram menos chances de ocorrer no período neonatal precoce.

\section{Discussão}

Os estudos sobre mortalidade são, em sua maioria, realizados com dados secundários. Em 1948, para garantir a qualidade desses dados, a Assembléia Mundial de Saúde recomendou um modelo de certificado médico de causa de morte. Desde 1950 , os campos para o preenchimento das causas do óbito estão padronizados, possibilitando que os coeficientes de mortalidade entre os diferentes países possam ser comparados 13,16. Os estudos sobre evitabilidade também utilizam dados secundários que, quer sejam de morbidade ou mortalidade, foram codificados pela CID-10 antes de serem categorizados em evitável e nãoevitável.

Este estudo foi realizado com dados secundários do SIM, fonte oficial de informações sobre mortalidade no país. Na série temporal utilizada, a causa básica do óbito foi codificada pela CID-10 e a observação do princípio geral e das regras de seleção da causa básica foi assegurada pelo programa de Seleção de Causa Básica que acompanha o programa do SIM.

Em Porto Alegre, os dados do SIM e SINASC são rotineiramente qualificados por meio de revisão de prontuários hospitalares, ambulatoriais e do Departamento Médico Legal. O PRÁ-VIVER realiza entrevistas com as mães sociais das crianças falecidas, procurando conhecer a história de vida da criança e de sua família. Por essa razão, dos 1.180 óbitos ocorridos entre 2000 e 2003, foi necessário excluir do estudo apenas 6 casos $(0,5 \%)$ por insuficiência de dados. Os outros 35 casos (3\%) foram excluídos por terem sua causa básica de óbito classificada como mal definida (P90-95, R00-99, exceto R-95). No ano 2000, a proporção dos óbitos infantis com causas mal definidas para os óbitos infantis em Porto Alegre foi inferior à do país, 12,3\%, e à da Região Sul, $5,2 \% 3$, mostrando a qualidade dos dados utilizados.

O delineamento de estudo de casos e controles possibilita verificar quantas vezes mais chances da ocorrência dos fatores em estudo na presença do desfecho, porém não permite o estabelecimento da causalidade. Entretanto, como o desfecho investigado é o óbito, pelo menos quatro dos critérios de causalidade de Hill são preenchidos: força de associação, temporalidade, plausibilidade e coerência 17,18 .

A literatura internacional dos últimos trinta anos sobre mortalidade evitável é vasta, mas, no que diz respeito à faixa etária específica que compreende todo o primeiro ano de vida, foram poucos os artigos encontrados nas bases de dados disponíveis, como também não foram localizados artigos que abordassem os fatores associados aos óbitos evitáveis. Desse modo, não foi possível comparar os resultados deste estudo com os de outros trabalhos similares. Por outro lado, a mortalidade infantil e seus determinantes são amplamente estudados e os óbitos infantis considerados, em sua maioria, como evitáveis. Por essa razão, utilizou-se artigos sobre fatores associados à mortalidade infantil para a comparação dos achados deste estudo.

A classificação de evitabilidade utilizada permitiu a identificação de $23,4 \%$ de óbitos nãoevitáveis. Esse achado é superior ao de outros trabalhos realizados nas regiões Nordeste 19,20 e Sudeste 21 , que encontraram, respectivamente, cerca de $20 \%$ e $13 \%$. A variação na proporção de óbitos não-evitáveis pode ser explicada pelo uso de diferentes classificações. Os critérios de evitabilidade não são definitivos e tampouco universais, dependendo dos avanços tecnológicos e de sua disponibilidade nos serviços de saúde 22 .

Nesse sentido, em relação à classificação de Ortiz ${ }^{12}$, observa-se que a síndrome do desconforto respiratório do recém-nascido ainda permanece entre as causas não-evitáveis, mesmo existindo medicação disponibilizada, inclusive nos hospitais conveniados ao SUS 23. Além disso, outras morbidades ainda permanecem como causas de óbitos não-evitáveis como as malformações congênitas graves, as anomalias e as síndromes cromossômicas, a despeito dos avanços na área da genética humana. No entanto, para muitas dessas doenças, a possibilidade de diagnóstico e intervenção precoces é, praticamente, inexistente na rede pública. À medida que a mortalidade evitável diminui, a proporção das mortes não-evitáveis tende a aumentar 24 . Não obstante, um país em desenvolvimento deve voltar seus esforços para as causas de óbitos que podem ser evitados.

Em relação aos fatores em estudo foram verificadas pequenas diferenças na magnitude das associações nas análises bivariadas e multivariadas. Isso sugere que o efeito dos fatores sobre o desfecho praticamente se dá sem a mediação das variáveis de hierarquias inferiores e sem a ocorrência de confundimento determinado pelas variáveis do mesmo nível e dos níveis superiores. 
Alguns autores 23,25 têm encontrado um maior risco de óbito para o sexo masculino no período neonatal, provavelmente relacionado ao amadurecimento pulmonar mais precoce no sexo feminino 23 . Foi também relatada uma maior freqüência de hospitalização para crianças do sexo masculino durante o primeiro ano de vida 26 . Embora, no presente estudo, tenha-se encontrado mais meninos entre os óbitos evitáveis do que entre os não-evitáveis, essa diferença não se mostrou significativa.

Quanto à escolaridade materna, esta variável tem sido utilizada como um indicador da condição sócio-econômica da mãe e de sua família, estando relacionada à qualidade dos cuidados com a saúde da criança 5. Estudos têm demonstrado que as populações com baixa inserção sócio-econômica e pouca escolaridade têm coeficientes de mortalidade infantil mais altos 25 . Neste trabalho, entre os casos, houve um maior número de mães com até três anos de escolaridade.

A associação entre idade materna e mortalidade infantil é bem conhecida. Mães adolescentes e desfechos desfavoráveis, tais como prematuridade, baixo peso ao nascer e maior taxa de parto cesárea parecem estar associados 27 . Entre as mães das crianças investigadas, $24,1 \%$ tinham menos de 20 anos de idade, porém não foi encontrada associação estatisticamente significativa com o desfecho.

A ausência de companheiro pode indicar uma maior vulnerabilidade social, uma lacuna no suporte emocional e econômico para a família. Alguns autores 28 relatam associação entre mortalidade neonatal e não ter o nome do pai informado na Declaração de Nascido Vivo. Outros autores 23 não puderam comprovar a influência do fato da mãe ser solteira no aumento da mortalidade neonatal, apesar da maior prevalência de baixo nível sócio-econômico. Entre os óbitos ocorridos por causas evitáveis encontrou-se menos mães sem companheiro, indicando um excesso significativo de mães solteiras entre os óbitos não-evitáveis.

A atenção à saúde da mãe e da criança tem sido apontada como fator de grande relevância para a redução da mortalidade infantil 5,6. As variáveis reprodutivas maternas e as relacionadas às condições de nascimento da criança permitem a avaliação da qualidade dessa atenção.

O número de filhos nascidos vivos está relacionado ao intervalo entre as gestações. Um intervalo menor que 24 meses tem sido considerado como um fator de risco para a mortalidade infantil 29. A OMS tem preconizado um intervalo interpartal superior a 36 meses, por favorecer o aleitamento materno e dar melhores condições à mãe no cuidado com a criança ${ }^{30}$. Em Porto Ale- gre, os dados do SINASC mostram um aumento percentual de mães primíparas, passando de $42,5 \%$ para $44,8 \%$ entre 2000 e 2003 e uma redução no percentual de mães com mais de três filhos nascidos vivos, de 15,3\% para 14,5\% (Secretaria Municipal de Saúde. Dados sobre nascidos vivos. http://lprweb.procempa.com.br/pmpa/ prefpoa/sms/usu_doc/sinasc_-_resultados.pdf, acessado em Jan/2005), acompanhando a tendência mundial de redução da fecundidade 28 . Alguns trabalhos relatam prevalência maior de óbitos infantis nas mães primíparas 27 e também nas grandes multíparas 28 . Neste estudo, porém, o número de filhos nascidos vivos e os óbitos evitáveis não se associaram.

As perdas fetais maternas têm sido associadas a uma maior prevalência de óbitos infantis 23,28 e a perdas fetais futuras 23 . Este estudo encontrou associação significativa entre ter filhos nascidos mortos e óbitos evitáveis. A gravidez múltipla, apontada como uma situação de risco para o óbito no primeiro ano de vida 21,31, não se mostrou associada aos óbitos evitáveis. Alguns autores observaram que não fazer o pré-natal também aumenta o risco para as perdas fetais 32 e óbitos infantis 28 , contudo não foi encontrada associação entre número de consultas de prénatal e óbitos evitáveis.

Em Porto Alegre, o SINASC registrou 19.197 crianças nascidas vivas no ano de 2003. Dessas, 237 apresentaram malformações congênitas, representando $1,2 \%$ do total ${ }^{32}$. A prevalência de malformações congênitas entre as crianças do estudo foi de $16,4 \%$, semelhante ao estudo sobre mortalidade perinatal realizado no Município de Fortaleza, Estado do Ceará, que encontrou $11,3 \% 33$. Neste estudo, a presença de malformação congênita esteve associada aos controles, isto é, à mortalidade não-evitável. Provavelmente, esse resultado se deve à classificação utilizada, em que as malformações mais graves fazem parte do grupo das causas de óbitos não-evitáveis.

O parto cesáreo apresentou associação significativa com os óbitos evitáveis, contudo, alguns trabalhos sobre a mortalidade infantil encontraram menor risco para óbito entre os nascidos vivos de parto cesáreo do que entre os nascidos vivos de parto vaginal 28,31.

Apesar da ampliação da oferta de serviços e de progressos na qualidade da assistência ao pré-natal e na atenção terciária ao recém-nascido, a prematuridade e o baixo peso ao nascer são ainda fatores de risco para a mortalidade infantil 23,28. Muitos dos fatores que concorrem para os partos prematuros são originados na mudança do estilo de vida nas últimas décadas, tais como o uso de álcool e fumo, estresse social e ocupacional, nutrição inadequada e outras 
condições em que as intervenções para uma gestação saudável deveriam ocorrer bem antes da concepção ${ }^{34}$. Nesta pesquisa, encontrou-se uma menor chance de prematuridade e baixo peso ao nascer entre os casos, isto é, ambas as categorias estiveram aumentadas entre os controles (óbitos não-evitáveis).

Não houve associação entre o local de nascimento e os óbitos evitáveis, possivelmente pelo pequeno número de nascimentos ocorridos fora do ambiente hospitalar. Do mesmo modo, o Apgar no 5 o minuto inferior a 4, utilizado na CID-10 como limite superior para definir asfixia neonatal grave 13 , não se associou aos óbitos evitáveis.

No período investigado, ocorreram mais óbitos neonatais do que pós-neonatais. Esse resultado está de acordo com o momento da transição epidemiológica da mortalidade infantil, evidenciando uma tendência de aumento do componente neonatal na mortalidade proporcional. No entanto, os óbitos evitáveis estiveram associados ao período pós-neonatal, e os não-evitáveis ao neonatal precoce. Isso se deve à classificação utilizada, na qual, as malformações congênitas graves e a membrana hialina

\section{Resumo}

Foi realizado um estudo de casos e controles para investigar a associação entre as categorias óbitos evitáveis e não-evitáveis e as variáveis sócio-demográficas, reprodutivas maternas e relacionadas às condições de nascimento da criança. Foram analisados 1.139 casos de óbitos infantis, ocorridos entre 2000 e 2003, com o auxílio da regressão logística multivariada, segundo modelo hierarquizado. As variáveis sexo, idade materna, número de filhos nascidos vivos, tipo de gravidez, local de nascimento e Apgar no 50 minuto não se associaram aos óbitos evitáveis. No entanto, a escolaridade materna $\leq 3$ anos ( $R C=1,56$; IC95\%: 1,01-2,45); mãe sem companheiro $(R C=0,65 ;$ IC95\%: $0,49-0,86)$ ou com filhos nascidos mortos (RC = 1,59; IC95\%: 1,012,48); ter malformação congênita $(R C=0,26$; IC95\%: 0,18-0,37); cesariana $(R C=1,52$; IC95\%: 1,10-2,11); idade gestacional entre 22 e 36 semanas $(R C=0,47$; IC95\%: 0,34-0,65); peso inferior a $2.500 \mathrm{~g}(R C=0,51$; IC95\%: 0,32-0,79) e ter menos de seis dias de vida $(R C=$ 0,62; IC95\%: 0,42-0,89) apresentaram associação significativa com os óbitos evitáveis. Esses achados podem contribuir na identificação de crianças em situação de maior vulnerabilidade.

Mortalidade Infantil; Fatores Epidemiológicos; Condições Sociais do recém-nascido estão incluídas na categoria óbito não-evitável.

Cabe ressaltar que a população em estudo é formada somente por crianças que foram a óbito, sendo classificado em evitável ou não-evitável. Desse modo, é possível afirmar que o comportamento de proteção, para óbitos evitáveis, apresentado pelas categorias não ter companheiro, presença de malformação congênita, prematuridade, baixo peso ao nascer e período neonatal precoce, na verdade, significa risco para a ocorrência de óbitos não-evitáveis. Por outro lado, a baixa escolaridade materna, a presença de filhos nascidos mortos e o parto cesáreo apresentaram comportamento de risco para os óbitos evitáveis, situações passíveis de intervenção por meio de políticas públicas sociais e da qualificação da atenção oferecida à gestante e ao recém-nascido.

As classificações de evitabilidade podem ser utilizadas como instrumentos de avaliação da qualidade da atenção à saúde. No entanto, seus critérios devem ser constantemente revisados, visando a incorporar os avanços tecnológicos, bem como, à disponibilização destes para os usuários do SUS.

\section{Colaboradores}

Ambos os autores participaram de todas as etapas de elaboração deste artigo. 


\section{Referências}

1. Oliveira L, Mendes M. Mortalidade infantil no Brasil: uma avaliação de tendências recentes. In: Minayo MC, organizador. Os muitos Brasis - saúde e população na década de 80. São Paulo: Editora Hucitec/ Rio de Janeiro: ABRASCO; 1999. p. 291-303.

2. Goldani MZ, Benatti R, Silva AA, Bettiol H, Correa JC, Tietzmann M, et al. Redução das desigualdades na mortalidade infantil na região Sul do Brasil. Rev Saúde Pública 2002; 36:478-83.

3. Ministério da Saúde. Indicadores de saúde. http:// tabnet.datasus.gov.br/cgi/mortinf/mibr.htm (acessado em Jan/2005).

4. Departamento de Análise de Situação de Saúde, Secretaria de Vigilância em Saúde, Ministério da Saúde. Dados e indicadores selecionados. Brasília: Ministério da Saúde; 2004.

5. Black RE, Morris SS, Bryce J. Where and why are 10 million children dying every year? Lancet 2003; 361:2226-34.

6. Victora CG, Wagstaff A, Schellenberg JA, Gwatkin D, Claeson M, Habiicht JP. Applying an equity lens to child health and mortality: more of the same is not enough. Lancet 2003; 362:233-41.

7. Rutstein DD, Berenberg W, Chalmers TC, Child CG, Fishman AP, Perrin EB. Measuring the quality of medical care, a clinical method. N Engl J Med 1976; 294:582-8.

8. Nolte E, Mckee M. Does healthcare save lives? Avoidable mortality revisited. London: The Nuffield Trust; 2004.

9. Charlton JR, Silver R, Hartley RM, Holland WW. Geographical variation in mortality from conditions amenable to medical intervention in England and Wales. Lancet 1983; 1:691-6.

10. Wigglesworth J. Classification of perinatal deaths. Soz Praventivmed 1994; 39:11-4.

11. Holland WW, editor. European community atlas of “avoidable death" 1985-89. Oxford: Oxford University Press; 1997.

12. Ortiz LP. Características da mortalidade neonatal no Estado de São Paulo [Tese de Doutorado]. São Paulo: Faculdade de Saúde Pública, Universidade de São Paulo; 1999.

13. Organização Mundial da Saúde. Classificação estatística internacional de doenças e problemas relacionados à saúde. 10a revisão. São Paulo: Centro Colaborador da OMS para a Classificação de Doenças em Português; 1995.

14. Victora CG, Huttly S, Fuchs S, Olinto MT. The role of conceptual frameworks in epidemiological analysis: a hierarquical approach. Int J Epidemiol 1997; 26:224-7.

15. Aerts D, Jobim R. The epidemiological profile of tuberculosis in southern Brazil in times of AIDS. Int J Tuberc Lung Dis 2004; 8:785-91.

16. Laurenti R, Mello-Jorge MH. O atestado de óbito. 3a Ed. São Paulo: Centro Brasileiro de Classificação de Doenças; 1996.

17. Fletcher RH, Fletcher SW, Wagner EH. Epidemiologia clínica: elementos essenciais. São Paulo: Editora Artmed; 2003.

18. Medronho RA, organizador. Epidemiologia. São Paulo: Editora Atheneu; 2003.
19. Vidal SA, Frias PG, Barreto FM, Vanderlei LC, Felisberto E. Óbitos infantis evitáveis em hospital de referência estadual do Nordeste brasileiro. Rev Bras Saúde Matern Infant 2003; 3:281-9.

20. Hartz ZM, Champagne F, Leal MC, Contandriopoulos AP. Mortalidade infantil "evitável" em duas cidades do Nordeste do Brasil: indicador de qualidade do sistema local de saúde. Rev Saúde Pública 1996; 30:310-8.

21. Gomes JO, Santo AH. Mortalidade infantil em município da região Centro-Oeste Paulista, Brasil, 1990 a 1992. Rev Saúde Pública 1997; 31:330-47.

22. Laloum D, Muller G, Speckel D. Avoidable perinatal mortality. Attemped evaluation and significance. Presse Med 1992; 21:1872.

23. Araujo BF, Bozetti MC, Tanaka AC. Mortalidade neonatal precoce no Município de Caxias do Sul: um estudo de coorte. J Pediatr (Rio J) 2000; 76:200-6.

24. Victora CG. Intervenções para reduzir a mortalidade infantil pré-escolar e materna no Brasil. Rev Bras Epidemiol 2001; 4:3-64.

25. Menezes AM, Barros FC, Victora CG, Tomasi E, Halpern R, Oliveira A. Fatores de risco para mortalidade perinatal em Pelotas, RS, 1993. Rev Saúde Pública 1998; 32:209-16.

26. Barros FC, Victora CG, Tomasi E, Horta B, Menezes AM, Cesar JA, et al. Saúde materno-infantil em Pelotas, Rio Grande do Sul, Brasil: principais conclusões da comparação dos estudos das coortes de 1982 e 1993. Cad Saúde Pública 1996; 12 Suppl 1:S87-92.

27. Ribeiro ER, Barbieri MA, Bettiol H, Silva AM. Comparação entre duas coortes de mães adolescentes em município do Sudeste do Brasil. Rev Saúde Pública 2000; 34:136-42.

28. Almeida MF, Novaes HM, Alencar GP, Rodrigues LC. Mortalidade neonatal no Município de São Paulo: influência do peso ao nascer e de fatores sociodemográficos e assistenciais. Rev Bras Epidemiol 2002; 5:93-107.

29. Rutstein SO. Factors associated with trends in infant and child mortality in developing countries during the 1990s. Bull World Health Organ 2000; 78:1256-70.

30. World Health Assembly. World Health Assembly resolution WHA47.5: infant and young child nutrition. Geneva: World Health Organization; 1994.

31. Morais Neto OL, Barros MB. Fatores de risco para mortalidade neonatal e pós-neonatal na região Centro-Oeste do Brasil: linkage entre bancos de dados de nascidos vivos e óbitos infantis. Cad Saúde Pública 2000; 16:477-85.

32. Victora CG, Barros FC, Vaughan JP. Epidemiologia da desigualdade. São Paulo: Editora Hucitec; 1988.

33. Leite AJ, Marcopito LF, Diniz RG, Silva AV, Souza LC, Borges JC, et al. Mortes perinatais no Município de Fortaleza, Ceará: o quanto é possível evitar? J Pediatr (Rio J) 1997; 73:388-94.

34. Wise PH. Efficacy and justice: The importance of medical research and tertiary care to social disparities in infant mortality. J Perinatol 1999; 19(6 Pt 2): S24-7.

Recebido em 01/Jun/2006

Versão final reapresentada em 13/Mar/2007

Aprovado em 01/Jun/2007 